\title{
Influencia de la recomendación 202 de la OIT en la legislación nacional de México: El caso del programa Prospera
}

\author{
Influence of the ILO recommendation 202 into the Mexican national legislation: \\ The Prospera program case
}

\author{
Gabriela Mendizábal \\ Universidad Autónoma del Estado de Morelos, México \\ Patricia Kurczyn \\ Instituto de Investigaciones Jurídicas, UNAM, México
}

\begin{abstract}
RESUMEN Desde la implementación de la Recomendación 202 de la OIT en el año 2012, cada país intenta de distintas formas dar cumplimiento a los pisos de protección social que ésta establece. Por ello, en este artículo se presenta el análisis de dicha implementación, tomando como base no los resultados en los beneficiarios - como es común-, sino a través del análisis en la legislación nacional de México de la incorporación de los nueve principios más importantes que la propia Recomendación establece, para diagnosticar si su adecuado cumplimiento permite establecer realmente un piso para la gente más pobre y si éste funciona como lo determina el instrumento internacional, en específico a través del análisis del programa Prospera.
\end{abstract}

PALABRAS CLAVE Pisos de protección social, programas sociales, protección social, principios de la Recomendación 202 de la OIT.

ABSTRACT Since the implementation of the ILO Recommendation 202 during 2012, each country has been trying in different ways to fulfill the social protection floors established in the Recommendation. Because of that, this article presents the analysis of each implementation, starting without the beneficiaries results - as is common-, but through the Mexican national legislation analysis of the incorporation about the nine most important principles of the Recommendation, with the objective to identify if the proper compliance allow the establishment of a truly social protection floor for the poorest people, and if this one works as the international instrument determines it. Specifically, through the analysis of the Prospera program. 
KEYWORDS Social protection floors, social programs, social protection, principles of the ILO Recommendation 202.

\section{Introducción}

En el año 2008, el secretario general de Naciones Unidas Ban Ki-Moon, con la finalidad de combatir el impacto social de la crisis económica del mismo año, se dio a la tarea de atender de manera inmediata este problema y buscar una solución para contrarrestar dicha crisis. Al respecto, en el año 2009 «se llegó a un acuerdo entre las agencias de la Organización de las Naciones Unidas para accionar nueve iniciativas conjuntas» (Naciones Unidas, 2016: 28). Una de ellas es la iniciativa del piso de protección social, la cual es abanderada por la Organización Internacional del Trabajo y la Organización Mundial de la Salud.

En el mismo año, la Organización de las Naciones Unidas impulsó la iniciativa del piso de protección social, que "considera la provisión de servicios esenciales y transferencias a todas aquellas personas en necesidad de protección para prevenir que caigan en estado de pobreza extrema o facilitar su salida de la misma» (Bertranou, 2010: 1), es decir, brindarles la protección social que les permita disfrutar su derecho a la seguridad social, estableciendo los mecanismos necesarios para otorgarles las garantías básicas de dicha rama del derecho, basadas en el acceso a un conjunto de bienes y servicios que constituyen la atención de salud esencial, seguridad básica del ingreso para los niños, seguridad básica del ingreso para las personas en edad activa que no puedan obtener ingresos suficientes y seguridad básica del ingreso para las personas en edad avanzada. ${ }^{1}$

Con motivo de dicha iniciativa propuesta por la ONU, en el año 2012 la Organización Internacional del Trabajo emitió la recomendación sobre los pisos de protección social número 202, donde orienta a los Estados miembros siguiendo los principios establecidos en la propia recomendación. ${ }^{2}$ El principal objetivo es llevar a cabo estrategias para ampliar la seguridad social aumentando progresivamente niveles más integrales de protección para el mayor número de personas, conforme a los sistemas nacionales de cada país.

Desde la implementación de la Recomendación 202, cada país intenta de distintas formas dar cumplimiento a los pisos de protección social (PPS) que ésta establece. Por ello, en este artículo se presenta el análisis de dicha implementación, tomando como base no los resultados en el acceso a prestaciones para los beneficiarios o los

\footnotetext{
1. «Socpro», Organización Internacional del Trabajo, disponible en http://bit.ly/2JYsdXw.

2. «La Recomendación sobre los pisos de protección social, 2012 (núm. 202)», Organización Internacional del Trabajo, disponible en http://bit.ly/2WnSBR3.
} 
cambios en los porcentajes en la matrícula escolar — como es común-, sino a través del análisis en la legislación nacional de México de la incorporación de algunos de los principios más importantes que la propia Recomendación establece: universalidad de la protección; adecuación y previsibilidad de las prestaciones; no discriminación; responsabilidad estatal; derecho a las prestaciones prescritas por la legislación nacional; solidaridad en la financiación; buen gobierno; coherencia con las políticas sociales, económicas y de empleo; organizaciones pertinentes y representativas de personas

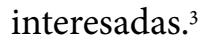

La hipótesis de esta investigación consiste en que muchos países -como México- ya contaban con programas asistenciales que adecuaron para dar cumplimiento a la Recomendación; sin embargo, ello no ha logrado que realmente cambie la calidad de vida de las personas, y aunque se pueda determinar que se está dando cumplimiento porque se adecuaron las normas jurídicas de los programas sociales, el nivel de la pobreza no ha disminuido y el porcentaje de aseguramiento no ha aumentado como se esperaba.

Es decir, a pesar de que existen datos contundentes de la entrega de beneficios y aumento en tasas de educación y salud, no se ha logrado fortalecer el vínculo entre educación y trabajo para tener un verdadero aseguramiento social, y por el momento son muy pocas las reformas tendientes a deslaboralizar la seguridad social y aumentar la cobertura de esta forma.

Por lo tanto, el presente artículo se compone de tres partes: la primera presenta la existencia de los programas sociales mexicanos previos a la Recomendación 202 que explica el contexto de la seguridad social en México, y permite comprender la problemática social en este país. La segunda analiza desde el punto de vista jurídico los principios incluidos en la citada recomendación de la OIT, para evaluar cómo se incorporan en el sistema, en qué medida se llevan a cabo y dónde surgen complicaciones para determinar si se aplican realmente en el marco de los pisos nacionales de protección social. Y, por último, la tercera parte se compone de las conclusiones y las fuentes de investigación respectivas.

\section{La protección social en México}

La protección social en México se ha caracterizado por el desarrollo de seguros sociales que protegen a trabajadores y sus familias dentro de la economía formal y una extensión de la cobertura mediante programas asistenciales tendientes a lograr dos objetivos.

3. Principios establecidos en el párrafo 3 de la Recomendación sobre los pisos de protección social, 2012 (núm. 202), 14 de junio de 2012, Ginebra. Relativo a que reconociendo la responsabilidad general y principal del Estado de poner en práctica la Recomendación, los miembros deberían aplicar los principios referidos. 
Primero, busca aliviar la pobreza de una gran parte de la población (43,6\% de la población total actualmente) (Coneval, 2016). ${ }^{4}$ Segundo, la inclusión en el otorgamiento de prestaciones a los trabajadores de la economía informal, que se ubican cerca del $57,7 \%$ de la población económicamente activa, ${ }^{5}$ desafortunadamente -como indica el porcentaje mencionado- sin lograrlo. Esto demuestra que al no contar con un verdadero sistema de seguridad social sostenible, tampoco se tiene uno de los elementos clave para la promoción de un crecimiento económico productivo con equidad que mitigue el impacto económico y social de las crisis económicas; es por ello que resulta tan importante la implementación de la Recomendación 202 de la OIT sobre los pisos de protección social, la cual se ve materializada mediante la ampliación de cobertura de los seguros sociales y la extensión del alcance los programas asistenciales.

Dentro de los programas sociales más emblemáticos se encuentra Prospera, que ha transitado a lo largo de varios años, cambiando de nombre y objetivos, según las expectativas y necesidades de cada gobierno en turno y en ocasiones aplicado con fines políticos, pero que ha mantenido la línea de las transferencias económicas y prestaciones para familias consistentes principalmente en ayuda alimentaria, becas escolares y atención básica en salud. Ahora, en el año 2019, el gobierno en turno amenaza con desaparecerlo, puesto que a pesar de los buenos resultados que ha tenido, ha sufrido drásticamente una reducción en el presupuesto asignado, como muestra la tabla 1.

No obstante lo anterior, para estar en posibilidad de comprender el entorno mexicano y la actual aplicación de los programas sociales que conforman el PPS, principalmente el programa Prospera, es necesario presentar brevemente dos partes del contexto nacional: la primera se centra en el desarrollo histórico de la seguridad social y los programas asistencialistas que son la base del actual Prospera; la segunda se centra en la problemática social que intenta solucionar dicho programa, como se resume a continuación.

\section{Protección social a través de seguros sociales y programas asistenciales}

A pesar de que desde 1952 la Organización Internacional del Trabajo adoptó el Convenio 102 sobre la seguridad social (norma mínima), es recién en 1961 que México lo ratifica. Cabe destacar que desde 1917, México había ya instaurado en la Constitución Política de los Estados Unidos Mexicanos los derechos sociales del trabajo y a la segu-

\footnotetext{
4. Cabe aclarar que Coneval hace una medición de la pobreza a nivel nacional, pero presenta datos actualizados cada dos años, la última medición fue en el año 2016 y presentó sus resultados en 2017.

5. "Indicadores de ocupación y empleo. Cifras oportunas durante febrero de 2019», INEGI, 27 de marzo de 2019, disponible en http://bit.ly/2IgL24L.
} 
Tabla 1. Presupuestos asignados

\begin{tabular}{|c|c|c|}
\hline Presupuesto asignado 2017 & Presupuesto asignado 2018 & Presupuesto asignado 2019 \\
\hline $\begin{array}{l}\$ 82.3 \text { millones } \\
\text { (US\$4.24 millones) }\end{array}$ & $\begin{array}{l}\$ 82,7 \text { millones } \\
\text { (US\$4.27 millones) }\end{array}$ & $\begin{array}{l}\$ 15,7 \text { millones } \\
\text { (US\$815.107) }\end{array}$ \\
\hline
\end{tabular}

ridad social, pero fue en 1925 cuando nace la Dirección General de Pensiones Civiles y de Retiro, la cual se transformaría años después (1960) en el Instituto de Seguridad y Servicios Sociales de los Trabajadores del Estado (ISSSTE), mismo que hasta la fecha otorga el aseguramiento social a los trabajadores al servicio del Estado (burócratas) a nivel federal; es decir, no incluye a los servidores públicos de las entidades federativas que quedan sujetos a sistemas estatales de seguridad social.

En 1926 se expide la Ley de Retiros y Pensiones del Ejército y la Armada Nacionales, que da origen al Instituto de Seguridad Social para las Fuerzas Armadas Mexicanas, el cual otorga seguridad social a los miembros de la Fuerza Armada y Marina Mexicana, así como a sus familias.

Por último, en 1943 se promulga la Ley del Seguro Social, con la cual surge el Instituto Mexicano del Seguro Social (IMSS) que desde entonces cubre las prestaciones correspondientes a los trabajadores de la iniciativa privada.

Mediante estos tres institutos que actualmente accionan a nivel nacional, más el Instituto del Fondo Nacional de la Vivienda para los Trabajadores (INFONAVIT), que solo responde a necesidades de vivienda y el establecimiento de los seguros sociales estatales antes mencionados, de cobertura particularizada a burócratas de la entidades federativas, se intentó extender la cobertura de forma horizontal a toda la población, pasando de proteger solo a trabajadores hasta llegar al núcleo familiar directo del trabajador, compuesto de manera general por los cónyuges, concubinas y concubinarios, hijos y ascendientes. ${ }^{6}$

Durante varias décadas, los seguros sociales crecieron también en cobertura vertical aumentando las prestaciones incluidas en cinco ramas de aseguramiento: riesgos de trabajo, enfermedades y maternidad, invalidez y vida, vejez, cesantía en edad avanzada y retiro y una atípica: guarderías y prestaciones sociales. No obstante, se debe señalar que se trata de esquemas contributivos que cubren a menos del $60 \%$ de la población y son regresivos. Sin embargo, con la crisis económica de los ochenta se empezó a dar mayor importancia a los programas asistenciales que se habían implementado, lo cual aunado a los cambios demográficos, al aumento de la migración

6. Cabe señalar que cada instituto tiene características y requisitos específicos para el otorgamiento de las prestaciones y que la atención a hijos se limita a cierta edad, mientras que para los ascendientes se condiciona a la dependencia económica. 
laboral, la precarización del empleo formal, el aumento de la pobreza y el desarrollo de nuevas enfermedades, entre otras causas, trajo como consecuencia que los seguros sociales detuvieran su desarrollo.

Cabe hacer mención que el marco normativo mexicano no establece una conceptualización de lo que es la seguridad social. Si bien es cierto en el artículo 123 apartado b) fracción 10 de la Constitución Política Mexicana (fuente normativa de la seguridad social en el país) establece la forma de organización de la seguridad social, no la define.

Por su parte, la Ley del Seguro Social establece en su artículo segundo que la finalidad de la seguridad social es garantizar el derecho a la salud, la asistencia médica, la protección de los medios de subsistencia y los servicios sociales necesarios para el bienestar individual y colectivo.

En ese contexto, podemos mencionar que México cuenta con un sistema de seguridad social híbrido: tanto bismarckiano a través de seguros sociales, como berveridgiano, pues el $40 \%$ de la población que no reúne los requisitos para ser asegurado en algún seguro social recibe prestaciones que otorgan los programas de naturaleza asistencial.

Dentro de los programas asistenciales ha sobresalido a nivel internacional Prospera, cuya característica principal es que se integra por tres componentes diagnosticados como de atención prioritaria para combatir la pobreza: ayuda para la alimentación, atención a la salud, educación y vinculación, porque es un programa que enlaza a los beneficiarios con el programa social que requieren para dar atención integral. Es un programa que surge desde 1973 con el nombre de Pider y se transforma en cada sexenio de gobierno, pero mantiene su esencia: la atención a la población en estado de vulnerabilidad por la pobreza. De esa forma el nombre cambió de Pider a Pronadri, Coplamar, Pronasol, Progresa, Oportunidades y actualmente Prospera.

De lo anterior, es posible resaltar que México fue pionero en establecer el derecho a la seguridad social para los trabajadores formales dentro de su Constitución, lo cual fue un logro para el constitucionalismo social, pero con el transcurso del tiempo ha sido un obstáculo en la incorporación de los trabajadores informales, los que han debido limitarse a la ayuda ofertada por los programas sociales creados o modificados por el gobierno en turno.

\section{Problemática social}

México es un país de grandes contrastes e inmensa riqueza cultural, donde la desigualdad social penetra en cada ámbito de cotidianidad y es necesario tener un contexto de datos estadísticos para poder comprender la necesidad del establecimiento de los pisos de protección social. 
En primer lugar, México es un país con una gran población. Según los datos del Instituto Nacional de Estadística, Geografía e Informática del cuarto cuatrimestre del 2018, México tiene 125.191.90o de habitantes con más de 31 millones de niños entre la edad de o y 14 años.7 En años recientes, la expectativa de vida de los mexicanos ha fluctuado: figuraba en 74,9 años en 2015, ${ }^{8}$ pero incrementó a 75,5 en 2018.

Del total de la población, 60.387.809 son hombres y 64.804.091 son mujeres. De este número total, $74,9 \%$ están en edad para trabajar, lo cual significa que más de 93 millones de personas tienen más de 15 años de edad. ${ }^{9}$

En segundo lugar, según el Consejo Nacional de Evaluación de la Política de Desarrollo Social, en 2016 (última medición multidimensional de Coneval) el 43,6 \% de los mexicanos (53,4 millones de personas), se encontraba en situación de pobreza y el $9,4 \%$ ( 9,3 millones) en pobreza extrema; ${ }^{10} 26,8 \%$ son vulnerables por carencia social de dos de los siguientes factores: educación, salud, seguridad social, vivienda, servicios básicos y alimentación; el 7\% son vulnerables por ingreso económico; y solo el 22,6\% es población no pobre y no vulnerable.

Esto indica que cerca del $59 \%$ de la población está ocupada sin seguridad social y el $48,9 \%$ de ellos gana solo hasta dos salarios mínimos. ${ }^{11}$

En tercer lugar, la informalidad laboral aún es un problema en México, que comprende más del 50\% de la población. De acuerdo con los datos de la Encuesta Nacional de Ocupación y Empleo de febrero de 2019, el 57,7\% de la población empleada tiene un empleo informal, mientras que la restante $42,3 \%$ tiene un empleo formal.

Por último, y al igual que en 2017, el dinero enviado por los migrantes (remesas) alcanzó los US $\$ 33.480$ millones en el 2018, lo que representa un incremento del 10,5\% en comparación al mismo año anterior. Esto significa que estas remesas sobrepasaron los ingresos de las exportaciones recibidas por petróleo (US\$26.510 millones de dólares). ${ }^{12}$

7. «Encuesta Nacional de Ocupación y Empleo (ENOE), población de 15 años y más de edad», INEGI, 16 de mayo de 2019, disponible en http://bit.ly/2XsaoEL.

8. «México en cifras», INEGI, disponible en http://bit.ly/2KAZ2t1.

9. «Encuesta Nacional», INEGI.

10. «Coneval informa los resultados de la medición de pobreza 2014», Coneval, 23 de julio de 2015, disponible en http://bit.ly/314vQ3j.

11. El salario mínimo en México para el 2019 es de $\$ 176,72$ pesos mexicanos diarios -equivalente a US $\$ 9,12-$ para la zona libre de la frontera norte y $\$ 102,68$ pesos mexicanos -equivalente a US $\$ 5,30-$ para el resto de la República.

12. «Valor de las exportaciones de petróleo crudo», Petróleos Mexicanos, disponible en http://bit. ly/2Xqk6pN. 


\section{Análisis de la incorporación de los principios de la Recomendación 202 en la legislación mexicana}

Si bien, como lo señala la OIT, la implementación del piso de protección social no implica la adquisición de nuevos derechos, sí reafirma y contribuye a la materialización del derecho humano a la seguridad social, consagrado como tal en la Declaración Universal de los Derechos Humanos (1948) y el Pacto Internacional de Derechos Económicos, Sociales y Culturales (1966) (Schwarzer y Casalí, 2010: 75).

La adecuación de los programas sociales existentes implica cambios en la legislación de cada país y México no es la excepción. La evaluación de la aplicación de los principios contenidos en la Recomendación 202 sobre los pisos de protección social en México ha tenido resultados favorables en las evaluaciones de impacto hechas por académicos de otros países, a las acciones sectorizadas en las que se implementó, desde antes de la propia Recomendación. Dentro de ellas sobresalen:

- De 2006 a 2012, la tasa global de fecundidad de mujeres entre 15 y 49 años disminuyó un $15 \%$ entre las beneficiarias del programa Oportunidades, mientras que en aquéllas que no son beneficiarias del programa la tasa aumentó en $10 \% .^{13}$

- Durante la aplicación del programa Oportunidades del 2000 a 2006, hubo una reducción de $11,8 \%$ en los casos de anemia en niños de menos de 2 años. ${ }^{14}$

- México recibió el galardón del ODM-1 por haber reducido la prevalencia de la subalimentación del 6,9\% de 1990-1992 al 4,6\% en 2012-2014..$^{15}$

- De 2012 a 2013, hubo en el programa Oportunidades una mejora en la inserción laboral de los jóvenes beneficiarios del programa, la cual se da a través del incremento en la escolaridad. Asimismo, se observó un aumento salarial de entre $12 \%$ y $14 \%$, aunque éste fue mayor para hombres que para mujeres. ${ }^{16}$

- Como resultado de las acciones de prevención del embarazo adolescente de septiembre 2014 a agosto de 2015, la campaña de salud sexual y planificación familiar en adolescentes del programa IMSS-Prospera llegó a 118.942 jóvenes en los 3.661 Centros de Atención Rural al Adolescente. ${ }^{17}$

13. «El programa Oportunidades ¿promueve los nacimientos?», Ensanut, disponible en http://bit. ly/2Zc5nPE.

14. «Un nuevo modelo de México para el mundo», Banco Mundial, 19 de noviembre de 2014, disponible en http://bit.ly/2EWqEVG.

15. «Reconoce la FAO a México por reducir la subalimentación un año antes de lo previsto», Gobierno de México, 30 de noviembre de 2014, disponible en http://bit.ly/2Ms2Yin.

16. «Informe de la evaluación específica de desempeño 2012-2013», Coneval, disponible en http://bit. ly/2XxjBtX.

17. «En \#3AñosDeGobierno un mejor futuro para las mujeres y las niñas», Gobierno de México, 1 de diciembre de 2015, disponible en http://bit.ly/2wyXF5Q. 
No obstante, es necesario analizar la forma en la que se adecuó hasta ahora la legislación nacional para dar cumplimiento a la Recomendación 202, en específico en relación con algunos de los principios más importantes para su ejecución.

\section{Responsabilidad del Estado}

$\mathrm{Al}$ analizar la fundamentación legal del programa Prospera, se puede afirmar que el gobierno mexicano es el proveedor de los beneficios y al mismo tiempo el administrador de los mismos. Por lo tanto, desde su propia normativa de creación se establece el papel del Estado a través de sus instituciones para implementar dicho programa, por supuesto sin la participación de empresas privadas.

La responsabilidad estatal se reconoce desde la Ley General de Desarrollo Social, una ley de aplicación en todo el territorio nacional, en la que menciona que uno de sus objetivos es «propiciar las condiciones que aseguren el disfrute de los derechos sociales y el acceso a los programas de desarrollo social y la igualdad de oportunidades» (Cámara de Diputados, 2016: 4), lo que se complementa con el Decreto de Creación de la Coordinación Nacional de Prospera, Programa de Inclusión Social, ${ }^{18}$ el 5 de septiembre de 2014, en el que se estipula que este programa coordinará y articulará las acciones de política social y programas ofertados entre las diferentes secretarías como las secretarías de Desarrollo Social, Economía, Hacienda y Crédito Público, Salud, Agricultura y la del Trabajo y Previsión Social, para lograr bajo esquemas de corresponsabilidad «mejorar la educación, la salud, la alimentación, la generación de ingresos» a la población que se encuentre en situación de pobreza extrema.

Ejemplo de ello es que las personas en extrema pobreza tienen «acceso prioritario a quince programas federales con salidas productivas, para incrementar su productividad y favorecer la generación de ingresos autónomos y sostenible». ${ }^{19}$

\section{Universalidad de la protección}

Lo primero que se debe señalar en el análisis del principio de la universalidad es que la cobertura universal en México es aún inexistente, lo que justifica y hace relevante la adopción de los pisos de protección social. De un lado tenemos el $40 \%$ de la población económicamente activa sin aseguramiento social, dentro de los que se han podido delimitar grupos sociales que se encuentran en estado de vulnerabilidad por no reunir los requisitos básicos que se establecen para incorporarse a un seguro social: ser trabajador de la economía formal o beneficiario de alguno de ellos; o contar con los me-

18. Decreto de Creación de la Coordinación Nacional de Prospera, Programa de Inclusión Social, publicado en el Diario Oficial de la Federación el 5 de septiembre de 2014.

19. «Preguntas frecuentes», Gobierno de México, 30 de marzo de 2016, disponible en http://bit. ly/2Xz5DYs. 
dios necesarios y la voluntad para incorporarse como profesionistas independientes al régimen obligatorio, aunque esto implica no tener derecho a todas las prestaciones.

Dentro de estos grupos sobresalen los trabajadores informales, los niños, los adultos mayores, las mujeres, los indígenas, los homosexuales, los migrantes, los presos y las personas con discapacidad. Las causas por las cuales cada uno de estos grupos están en estado de vulnerabilidad es diferente: por ejemplo, las mujeres por discriminación de género en el acceso al trabajo formal o al deber encargase del cuidado de niños, adultos mayores, enfermos y personas con discapacidad; los reos porque en México no hay una regulación que les brinde prestaciones de seguridad social, y a pesar de que algunos hacen trabajos para empresas privadas desde los centros de reclusión, no cotizan para las pensiones.

Para los niños en específico, el programa Prospera otorga, en educación, becas educativas a menores de dieciocho años integrantes de las familias beneficiarias que cursen y concluyan sus estudios de educación básica y media superior; apoyo para la adquisición de útiles escolares; apoyo para jóvenes con Prospera; acceso a exbecarios para garantizar su continuidad educativa a estudios de nivel superior; becas para estudios de nivel superior y para jóvenes con discapacidad y nuevas modalidades educativas como la no escolarizada, educación técnica y formación para el trabajo.

En alimentación, se otorgan beneficios económicos mensuales con la finalidad de contribuir a la compra y diversificación de sus alimentos y beneficios en especie, además de: i) promoción de la nutrición y alimentación saludable para los niños y las niñas menores de cinco años, las mujeres embarazadas y en periodo de lactancia; ii) suplementos alimenticios para complementar su dieta diaria, y acceso a comedores comunitarios del programa denominado la Cruzada Nacional contra el Hambre; y iii) la Estrategia Integral de Atención a la Nutrición (ESIAN) que atiende problemas de salud y nutrición de forma integral y con énfasis en los primeros mil días del individuo (desde el embarazo hasta los dos años).

En salud, ofrece protección sanitaria integral a través de dos programas: i) el seguro médico Siglo XXI, que otorga atención a la salud a través del IMSS, que es el seguro social público obligatorio, con mayor cobertura en el país, para todos los menores de cinco años; y ii) la atención básica en salud mediante el Seguro Popular de Salud. ${ }^{20}$

Sobre los trabajadores informales, se puede resaltar que tanto el decreto por el cual se crea la Coordinación Nacional de Prospera, como las reglas de operación del mismo para el ejercicio fiscal 2016, señalan como parte de sus objetivos la generación de ingreso, capacitación e inclusión laboral en el mercado formal que incrementen las capacidades productivas de las familias beneficiarias y sus integrantes para mejorar su bienestar económico, por lo que se entiende que la atención a los trabajadores informales se reduce a la capacitación para el empleo, la atención con servicios bási-

20. Se trata de un programa asistencial, a pesar de que lleva el nombre de «seguro». 
cos de salud a través del Seguro Popular de Salud y un apoyo monetario mensual a las personas mayores, integrantes de las familias beneficiarias, con una edad de 70 años o más, quienes fueron previamente identificados en este grupo. Este apoyo se otorga en tanto los beneficiarios no sean atendidos por el Programa de Pensión para Adultos Mayores de la Sedesol. ${ }^{21}$

Para los migrantes el programa contempla todas las prestaciones, siempre y cuando tengan como familia un ingreso mensual per cápita estimado menor a la línea de bienestar mínimo ajustada (LBMa) y comprueben su legal estancia mediante los documentos migratorios correspondientes. ${ }^{22}$

Como se ha podido observar, el programa Prospera se ha ajustado para dar seguimiento a la Recomendación 202. No obstante, en específico en la cobertura universal, si bien tiende a la realización progresiva, no incluye objetivos y plazos específicos para su logro que permitan suponer que se alcanzará el 100\% de población cubierta en tiempo determinado o tampoco establece cómo avanzará en la cobertura para dar el paso de un piso de protección esencial mediante el asistencialismo al aumento progresivo del aseguramiento social. Esto es conveniente para el gobierno, porque no tiene obligaciones determinadas que al ser cuantificables impliquen obligaciones específicas, cuyo cumplimiento pueda serles demandable.

\section{Derecho a las prestaciones prescritas por la legislación nacional}

Cada uno de los programas que coordina Prospera establece quiénes serán sus beneficiarios y qué requisitos deben cumplir, lo cual se inicia con una encuesta socioeconómica, cuyas respuestas por parte de la familia determinan si pueden ser beneficiarios; sin embargo, al analizar los derechos a las prestaciones basados en la ley se deben atender tres cuestiones.

En primer lugar, que el marco legal mexicano se integra de la siguiente manera: i) el bloque de constitucionalidad y convencionalidad que está compuesto por la Constitución Política de los Estados Unidos Mexicanos y a partir del año 2011 con los tratados internacionales ratificados en materia de derechos humanos, que no contravengan la norma magna, con la intención de ofrecer a las personas la protección más amplia; ii) los tratados internacionales en general que México haya firmado con la aprobación del Senado de la República; iii) encuentran las leyes generales y las de carácter federal, leyes orgánicas y especiales, las Constituciones de cada Estado y leyes estatales; y iv) los reglamentos y las normas individualizadas.

21. De acuerdo con las reglas de operación de Prospera, para el ejercicio fiscal 2016, publicadas en el Diario Oficial de la Federación el 30 de diciembre de 2015.

22. Acuerdo por el que se emiten las Reglas de Operación de Prospera Programa de Inclusión Social, para el ejercicio fiscal 2016. 
En segundo lugar, una característica general de los programas asistenciales es que carecen de mecanismos directos de exigibilidad jurídica (recursos administrativos). En tercer lugar, y no obstante lo anterior, existen mecanismos generales de justiciabilidad que pueden ser aplicables:

- Juicio contencioso administrativo o juicio de nulidad..$^{23}$

- Juicio de amparo. ${ }^{24}$

- Quejas ante comisiones de derechos humanos.

- Juicio civil por reparación del daño.

En otras palabras, a pesar de que la asistencia social pública no genera un derecho objetivo (García Máynez, 2008: 36 ), ${ }^{25}$ existe la posibilidad jurídica de entablar un juicio de amparo, civil o administrativo para reclamar el incumplimiento del otorgamiento de las prestaciones asistenciales ligadas a derechos humanos. No obstante, esto genera gastos por los honorarios de abogados, trámites y más, y estamos en presencia de beneficiarios, que son personas en estado de vulnerabilidad social, primero por estar en situación de pobreza y pobreza extrema, y segundo por su falta de acceso a niveles de educación media y superior, lo que en sí mismo hace casi imposible que demanden la justicia para acceder a los beneficios de programas asistenciales.

Sobre el tema de la corrupción, se puede mencionar que, debido a ello - entre otros factores-, dichos programas asistenciales con regularidad son utilizados con fines políticos que van desde dar una buena imagen pública de los gobernantes en turno hacia los gobernados, hasta para lograr obtención de votos de los ciudadanos en tiempos electorales. Aun así, el programa Prospera establece una pequeña válvula de escape en dos vertientes que sirven para que el beneficiario manifieste su inconformidad con el mismo: i) quejas y denuncias y ii) el derecho de audiencia, aunque se debe señalar que se trata únicamente de figuras cercanas al buzón de quejas y sugerencias.

Desafortunadamente, los recursos que establecen las reglas operacionales primeramente no son mecanismos directos de impugnación que garanticen una celeridad al resolverlos; segundo, estos medios de impugnación no suelen ser eficaces y accesi-

23. Es aquel juicio que se interpone contra las resoluciones administrativas definitivas que establece la Ley Orgánica del Tribunal Federal de Justicia Fiscal y Administrativa, cuando se desee impugnar un acto de autoridad que ha afectado al particular o a la autoridad misma.

24. Artículos 103 y 107 constitucional. Es un medio procesal constitucional del ordenamiento jurídico mexicano que tiene por objeto específico proteger los derechos humanos establecidos en la Constitución, así como en los tratados internaciones de los que México sea parte de los actos de todas las autoridades nacionales cuando éstos violen los referidos derechos fundamentales y sus garantías.

25. El derecho objetivo es un conjunto de normas. Se trata de preceptos imperativo-atributivos, es decir, de reglas que, además de imponer deberes, conceden facultades. 
bles para los beneficiarios, y por ende suelen ser costosos para poder interponerlos; y tercero, al estar plasmados en las reglas operacionales pueden variar año a año, lo que no garantiza la estabilidad o la variación del procedimiento cuando el ejecutivo federal lo determine.

\section{Adecuación y previsibilidad de las prestaciones}

Sobre la adecuación se puede decir que Prospera es un programa que se adecúa a la Recomendación 202, ya que es específico para la atención a personas vulnerables.

$\mathrm{Al}$ respecto, sus reglas de operación sostienen que los subsidios para el desarrollo social de Prospera se destinarán, en las entidades federativas, exclusivamente a la población en condiciones de pobreza, de vulnerabilidad, rezago y de marginación, de acuerdo con los resultados que defina el Consejo Nacional de Población (Conapo) y a las evaluaciones del Consejo Nacional de Evaluación de la Política de Desarrollo Social (Coneval) en los programas que resulte aplicable, y la Declaratoria de Zonas de Atención Prioritaria formulada por la Cámara de Diputados, mediante acciones que promuevan la superación de la pobreza a través de la educación, la salud, la alimentación, la generación de empleo e ingreso, autoempleo y capacitación; protección social y programas asistenciales. ${ }^{26} \mathrm{Al}$ respecto, el Coneval determinó para la medición de la pobreza la línea de bienestar mínimo, ${ }^{27}$ que es equivalente al valor de la canasta alimentaria (Coneval, 2016) por persona al mes: ${ }^{28}$ en el ámbito urbano, para el 2019 corresponde a $\$ 1.554,12$ pesos mexicanos (US $\$ 80,23$ ), y en el ámbito rural a $\$ 1.103$,01 pesos mexicanos $(\mathrm{US} \$ 56,94){ }^{29}$

26. Acuerdo por el que se emiten las Reglas de Operación de Prospera Programa de Inclusión Social, para el ejercicio fiscal 2015.

27. «Medición de la pobreza: Preguntas frecuentes», Coneval, disponible en http://bit.ly/2ZSPfmF. La línea de bienestar mínimo se fundamenta en la propuesta metodológica de la Comisión Económica para América Latina y el Caribe (Cepal), que toma como referencia los patrones de gasto de los hogares para aproximarse a los valores de consumo de energía y micronutrientes de acuerdo con diferentes niveles de ingreso y sirve de base junto con los índices de pobreza para establecer las medidas y acciones de la política social implementada a nivel nacional.

28. Es importante observar que esta canasta se forma a partir de la información de la Encuesta Nacional de Ingresos y Gastos de los Hogares, del coeficiente de adecuación energética y los ingresos de los hogares. De esta manera, la canasta alimentaria no solo se apega al patrón de consumo, sino también a las normas de nutrición adecuada. Una vez que se tiene el listado de productos y las cantidades de los alimentos que componen la canasta, su valor es igual al gasto reportado por el estrato de referencia. $\mathrm{Su}$ valor se actualiza a partir de los índices de precios de cada alimento reportado por INEGI para el año en cuestión.

29. «Medición de la pobreza 2008-2016: Evolución de las líneas de pobreza por ingresos», Coneval, disponible en http://bit.ly/2wHD5QF. 
Derivado de este índice de pobreza es que para identificar las familias beneficiarias se efectúa una encuesta de información socioeconómica y demográfica muy sencilla que el jefe o jefa de familia deben contestar, sin necesidad de comprobar la información proporcionada. Esto agiliza su incorporación, pero, aunque los documentos que se solicitan son muy simples, en ocasiones las personas por su estado de vulnerabilidad social no pueden proporcionar ni siquiera su acta de nacimiento y no hay un mecanismo del programa que les auxilie en ello, lo que les impide recibir beneficios.

Como se mencionó en apartados anteriores, en el año 2012 - año de la iniciativa de PPS- la población total mexicana en pobreza era de 55,3 millones, lo que representaba el $45,2 \%$ del total de la población nacional. La última medición oficial data del 2016 e indicaba a 53,4 millones de personas en pobreza y 9,3 millones en pobreza extrema, y no se cuenta con proyecciones serias sobre el tema. Sin embargo, se considera que si México lograse mantener un crecimiento económico por persona del $2 \%$ anual, le tomaría 60 años eliminar la pobreza extrema, mientras que si creciera $3 \%$ por persona, tardaría 40 años en abatir este rezago. Para esquematizar mejor la problemática, se puede ejemplificar que en el 2014 el crecimiento fue de $2,1 \%,{ }^{30}$ mientras que en 2015 fue del $2,5 \% .{ }^{31}$

La previsibilidad no es una de las características principales del Prospera, pero a pesar de ello se cuentan con prestaciones tanto preventivas como remediales. Dentro de las primeras se encuentra el componente de salud, que permite el acceso al paquete básico garantizado de salud y la ampliación progresiva a las 27 intervenciones de salud pública del CAUSES a las familias beneficiarias, con el propósito de impulsar el uso de los servicios de salud preventivos y el autocuidado de la salud y nutrición de todos sus integrantes, con énfasis en la población más vulnerable, como niños y niñas, mujeres embarazadas y en periodo de lactancia.. ${ }^{32}$

Un ejemplo más de las líneas del programa se encuentra encaminada a la prevención social de la violencia y la delincuencia establecido en el numeral 3.1.2 de las reglas de operación del programa, cuyas acciones tienen por objeto atender los factores de riesgo y de protección vinculados a la violencia y la delincuencia.

30. «INEGI estima que el PIB creció 2,2\% anual en 4T», El Economista, 31 de enero de 2017, disponible en http://bit.ly/2K2zUft.

31. «México crece 2,5\% en 2015», El Financiero, 29 de enero de 2016, disponible en http://bit.ly/3156G4R.

32. Acuerdo por el que se emiten las Reglas de Operación de Prospera Programa de Inclusión Social, para el ejercicio fiscal 2015. 
Por su parte, como ejemplo de las prestaciones remediales se otorga la pensión a las personas de la tercera edad, ${ }^{33}$ que consiste en un apoyo económico para quienes durante su vida productiva no contaron con un trabajo formal. ${ }^{34}$

\section{No discriminación}

La no discriminación se incluye en las normas básicas del Prospera. De forma particular resalta el punto 11, «Enfoque de derechos las reglas de operación», que establece:

Se fomentará que las y los servidores públicos involucrados en la operación del Programa, promuevan, respeten, protejan y garanticen el ejercicio efectivo de los derechos humanos de las y los beneficiarios, de conformidad con los principios de universalidad, interdependencia, indivisibilidad y progresividad, brindando en todo momento un trato digno y de respeto a la población objetivo, con apego a los criterios de igualdad y no discriminación.

Dentro del programa cobran importancia las acciones de discriminación positiva que otorgan beneficios preferentemente a mujeres, destacando dentro de ellas las siguientes:

- En el área de becas, los montos de los apoyos educativos son mayores para las mujeres, con el objetivo de contribuir a disminuir la brecha de género en materia educativa. ${ }^{35}$ Esto ha traído resultados medibles, como que las mujeres jóvenes beneficiarias de Prospera fueron en promedio o,65 años más a la escuela, o casi ocho meses, con lo que aumentaron sus niveles de escolaridad en las últimas décadas. La escolaridad promedio de las mujeres de quince años y más pasó de 6,2 a 8,5 años de estudios entre 1990 y 2010, mientras que la de los hombres pasó de 6,8 a 8,8 años en el mismo lapso. Las diferencias también se acortaron: por arriba del promedio nacional destaca la escolaridad de las mujeres entre 15 y 24 años, la cual superó la de los hombres al pasar de 7,9 a 10,2 años, entre 1990 y 2012; mientras que la de los varones subió de 7,9 a 9,9 años en el mismo lapso (Moctezuma Navarro, Narro Robles y Orozco Hernández, 2014).

- El programa Seguro de Vida para Jefas de Familia brinda a hijas e hijos en estado de orfandad materna, de hasta 23 años, apoyos económicos.$^{36}$

33. Como resultado se tiene que en el tercer bimestre del 2015 se apoyaron a 5.216.809 adultos mayores de 65 años con un total de $\$ 1.160$ pesos mexicanos a cada uno, de forma bimestral (US\$59,88) mediante el programa Pensión para Adultos.

34. «Programa de pensión para adultos», Secretaría de Biemestar, disponible en http://bit.ly/2LanUZP

35. «Componente prospera educación», Gobierno de México, disponible en http://bit.ly/2JoojCN.

36. Durante el primer trimestre del 2016 hubo 14, 520 jefas de familia que fallecieron. 
- El Programa de Estancias Infantiles busca apoyar a madres trabajadoras, es decir, aquellas madres que trabajan, buscan empleo o estudian. ${ }^{37}$

- Se incorporan de manera prioritaria a quince programas de Sedesol para lograr el empoderamiento a través de la iniciación de proyectos propios u opciones para trabajar. ${ }^{38}$

- El Programa de Apoyo a las Instancias de Mujeres en las Entidades Federativas contribuye a una sociedad igualitaria mediante la prevención y atención de la violencia contra las mujeres. ${ }^{39}$

Como se puede observar, este paquete de prestaciones en especie y beneficios económicos otorgado a las mujeres constituye una acción afirmativa en contra de la discriminación de género. De forma que los cambios implementados en el programa, además de empoderar a la mujer dentro de sus familias al tener un ingreso por el hecho de ser mujer, poco a poco, a través de los programas de inclusión productiva y laboral, se espera que permitan cerrar la brecha de desigualdad tan grande entre hombres y mujeres, en el papel de la mujer como cuidadora de niños, enfermos, ancianos y personas con discapacidad, lo que ha tenido como consecuencia reducir sus posibilidades de incorporarse al mercado formal de trabajo y legitimó al padre a distanciarse de las tareas domésticas y familiares.

\section{Solidaridad en la financiación}

La solidaridad financiera está ausente en las normas que regulan el prospera, de forma tal que mientras los seguros sociales públicos se financian tripartitamente (Estado, trabajador y patrón), los beneficios que otorga Prospera se financian directamente del presupuesto anual asignado por el presidente de la República en turno y avalado por el Congreso de la Unión. De esta manera, la solidaridad de la carga financiera no se distribuye entre generaciones o personas sanas y enfermas, sino que depende de los impuestos generales al consumo y al ingreso, por lo que los trabajadores de la economía formal conllevan una doble tributación para sostener sus seguros sociales y la carga financiera de las prestaciones asistencialistas. Si se toma en cuenta que, de los trabajadores formales afiliados ante el IMSS, el 73,9\% gana entre 1 y 3 salarios mínimos, el 14,7\% gana entre 4 y 5 salarios mínimos, mientras que

37. «Programa de Estancias Infantiles para Apoyar a Madres Trabajadoras», Gobierno de México, 30 de noviembre de 2015, disponible en http://bit.ly/2WUiI1E.

38. «Lineamientos operativos de Prospera Programa de Inclusión Social para 2015», Prospera, 4 de agosto de 2015, disponible en http://bit.ly/2FyliNI.

39. «Programa de Apoyo a las Instancias de Mujeres en las Entidades Federativas (PAIMEF)», Instituto Nacional de Desarrollo Social, Ciudad de México, 12 de julio de 2016, disponible en http://bit. ly/3opZE9z 
solo el 5\% gana entre 6 y 12 salarios mínimos, ${ }^{40}$ es comprensible la pobreza en gran parte de la población.

\section{Buen gobierno}

El Prospera es un programa que eminentemente coordina la atención a grupos vulnerables entre diferentes Secretarías de Estado y sus programas. Por ello, va encaminado a la gestión y administración del régimen de protección social para las personas que no están aseguradas socialmente. Sin embargo, mediante evaluación del Coneval se estableció que aún se requieren elementos de coordinación más efectivos en beneficio de las familias. ${ }^{41}$

En la normativa operacional de Prospera se hace mención en su parte introductoria que las reglas de operación tienen, entre otros objetivos, asegurar una aplicación eficiente, eficaz y transparente de los recursos públicos destinados a su operación. En cuanto a los criterios indispensables de evaluación para contribuir al cumplimiento del principio de un buen gobierno, las referidas reglas incluyen en el punto 6 los criterios para establecer evaluaciones que coadyuven a la rendición de cuentas transparente en el manejo de los recursos, determinando las formas y periodos en que se van a llevar dichas evaluaciones enfocadas a los recursos ejercidos, las acciones efectuadas y las metas logradas entre otras. Por su parte, el punto 8, «Seguimiento operativo, control y auditoría», refiere que se aplicará un modelo de seguimiento operativo convenido conjuntamente con los sectores involucrados.

Como se puede observar, desde la base jurídica sí existen elementos que logren ver un intento de transparentar la aplicación de los recursos financieros y el impacto de las prestaciones que se otorgan; pero aún hoy se requiere desarrollar una agenda de evaluación integral que se enfoque particularmente en los aspectos prioritarios que permitan a la población salir de la pobreza y dar el paso hacia el aseguramiento social, con un cronograma que permita la progresividad coherente y medible en el logro de los objetivos, porque las evaluaciones solo miden logros alcanzados sin tener un parámetro de alcance de objetivos medibles.

$\mathrm{Al}$ respecto, se puede señalar que no en cada programa se cuentan con informes financieros y actuariales, pero sobresalen los que proporciona IMSS-Prospera 2015 (IMSS, 2015), por tratarse de un instituto del seguro social que tiene características diferentes a los programas asistencialistas. Sin embargo, existe la obligación de rendir cuentas y, en su caso, la información que se oculte puede solicitarse a través del Insti-

40. Ana Karen García, «¿Cuánto ganan los mexicanos?», El Economista, 24 de julio de 2018, disponible en http://bit.ly/317G3w2.

41. «Evaluaciones Específicas: Evaluaciones específicas de desempeño 2014-2015», Coneval, disponible en http://bit.ly/2ZhSoMv. 
tuto Nacional de Transparencia, Acceso a la Información y Protección de Datos Personales (INAI), que podrá imponer sanciones a aquellas dependencias y organismos que se nieguen a dar cuentas de sus planes, programas, financiamiento y resultados, en los términos de las Leyes General ${ }^{42}$ y Federal ${ }^{43}$ de Transparencia y Acceso a la Información Pública (LGTAIP y LFTAIP).

Diversos estudios coinciden en la problemática común de los programas asistenciales como Prospera: falta de transparencia en los trámites, en la gestión de los recursos y en el otorgamiento de los apoyos; actos irregulares y de corrupción por parte de servidores públicos y nula exigibilidad del cumplimiento de su obligaciones a los servidores públicos que ejecutan los programas; uso electoral de los programas sociales; así como pocos informes financieros y actuariales (Centro de Estudios Sociales y de Opinión Pública, 2014: 4).

\section{Coherencia con las políticas sociales, económicas y de empleo}

La coherencia de políticas con el desarrollo del país, y en especial con el combate a la pobreza, abarca desde el diseño hasta la implementación y la evaluación de las políticas públicas de los diferentes actores políticos, lo cual - como ha quedado de manifiesto- se incluye en normas jurídicas expedidas por el Congreso de la Unión y decretos aprobados por el Ejecutivo, que además son acordes al Plan Nacional de Desarrollo vigente (2012-2018).

El marco jurídico de referencia en materia de política social toma en consideración cuatro puntos importantes para la coherencia.

En primer lugar, la determinación de los grupos vulnerables en torno a la pobreza en el país, pero además atiende la división etaria que la Recomendación 202 establece en niños, adultos y ancianos. También se incluyen programas y prestaciones con perspectiva de género, es decir, los beneficios se otorgan a grupos vulnerables focalizados

En segundo lugar, existen incentivos fiscales que contribuyen a la creación de empleo y al aseguramiento social de grupos en estado de vulnerabilidad, por ejemplo: i) se otorgará un estímulo fiscal a quien contrate adultos mayores, consistentes en el equivalente al $25 \%$ del salario efectivamente pagado a las personas de 65 años y más; ii) el patrón que contrate a personas que padezcan discapacidad motriz $-\mathrm{y}$ que para superarla requieran usar permanentemente prótesis, muletas o sillas de ruedas-, mental, auditiva o de lenguaje en un $80 \%$ o más de la capacidad normal o tratándose

42. Ley General de Transparencia y Acceso a la Información Pública, publicada en el Diario Oficial de la Federación el 4 de mayo de 2015.

43. Ley Federal de Transparencia y Acceso a la Información Pública, publicada en el Diario Oficial de la Federación el 9 de mayo de 2016. 
de invidentes, podrá deducir de sus ingresos, un monto equivalente al 100\% del impuesto sobre la renta de estos trabajadores, siempre y cuando el patrón cumpla con la obligación de darlos de alta ante el Instituto Mexicano del Seguro Social (Ley del Impuesto Sobre la Renta, artículo 186).

Como tercer punto, en educación se otorgan una gran cantidad de becas que van desde los programas de Prospera hasta programas estatales, como beca salario en el estado de Morelos, becas de educación superior y posgrado como la que otorga el Consejo Nacional de Tecnología (Conacyt), etcétera. Desafortunadamente, un punto bastante cuestionable es la falta concreta de políticas de vinculación entre educación y mercado de trabajo.

Por último, en materia de empleo, Prospera incluye quince programas productivos, ${ }^{44}$ pero la mayoría van encaminados al autoempleo, que no reúne «por mucho» las características del trabajo decente y, aunque contribuye al ingreso familiar, no servirá para volver formales a los trabajadores informales.

Algunos ejemplos de coherencia en las políticas públicas de fomento al empleo, que se pueden citar son que, en el año 2015, el Servicio Nacional del Empleo brindó a 50.000 jóvenes de Prospera servicios gratuitos de vinculación productiva como asesoría, orientación y canalización a vacantes: «A éstos se sumarán 150.000 anualmente, beneficiando durante este sexenio a un total de 650.000 jóvenes que recibirán servicios que les permitan incorporarse al mercado laboral formal».45 Pese a la información anterior, no existen datos oficiales de cuántos empleos se han ocupado y con continuidad en los mismos gracias a este programa. Además, en materia de autoempleo se pueden citar los microcréditos para establecer principalmente pequeñas empresas. ${ }^{46}$

\section{Conclusiones}

Lo primero a destacar en este análisis es que sí se cuenta con instrumentos jurídicos que den sustento a Prospera y los programas que vincula, como base a la protección social para grupos sociales en estado de vulnerabilidad, con el fin de acercase lo más posible a los objetivos de la Recomendación 202. Por otra parte, es sumamente lamentable que los índices de pobreza en las últimas décadas se mantengan constantes,

44. «Lineamientos objetivos», Prospera.

45. «Es prospera un programa social de vanguardia a nivel mundial», Programa de Inclusión Social, 10 de febrero de 2015, disponible en http://bit.ly/2 $\mathrm{XXD}_{3} \mathrm{U}_{2}$.

46. Un ejemplo de autoempleo es el que se da en San Andrés Tuxtla en Veracruz, en el que se invirtieron 24 millones de pesos mexicanos para apoyar 789 proyectos en 51 localidades. Dentro de estas localidades se destaca la producción de estevia en huertos familiares, en donde el $90 \%$ de los titulares son mujeres inscritas en el programa Prospera y obtienen un ingreso mensual de hasta $\$ 10.000$ pesos mexicanos. 
lo que nos lleva a cuestionarnos el uso de los recursos públicos para supuestamente reducirlos.

De un lado se observa una mejora en la calidad de vida de miles de mexicanos. De otro lado, no se ha logrado cortar la dependencia de estos programas para mantener una mejor calidad de vida, puesto que siguen siendo personas en pobreza y pobreza extrema, que sin las transferencias y prestaciones gubernamentales perecerían.

Dado lo anterior, puede señalarse que los resultados del programa Prospera y los quince programas que éste vincula, en cuanto a la extensión de la cobertura en materia de seguridad social que los pisos de protección social deben dar a los grupos sociales en estado de vulnerabilidad, como los trabajadores informales, no ha sido resuelta. Sin embargo, el viraje que el actual gobierno ha dado al programa debería conducir a la transición de las prestaciones asistencialistas dedicadas a mejorar la calidad de vida momentánea, hacia la incorporación al mercado formal del trabajo y con ello al acceso de las prestaciones de seguridad social del segundo escalón de la seguridad social: los seguros sociales.

En ese sentido, resultan cuestionables las políticas desarrolladas para el fortalecimiento del empleo formal que lejos de disminuir va en aumento a cada año.

Dentro de las conclusiones más importantes, se pueden señalar, primero, que algunas de las aristas de la problemática social en México son: una etapa pronunciada de lento crecimiento económico, persistencia de la pobreza y la desigualdad, incremento de los problemas de inseguridad, falta de credibilidad en las instituciones públicas - debido a la corrupción-y grandes flujos migratorios (Murayama Rendón y Gómez Tovar, 2015: 89). Esto justifica la implementación de los pisos de protección social, en específico para los trabajadores informales, y aunque se contempla para ellos la inclusión laboral en el mercado formal, a través del incremento de sus capacidades productivas, hoy en día solo es medible la mejora de su bienestar económico, no así el aumento de la cobertura para llegar al aseguramiento social.

Al respecto, se debe señalar que con ello se estaría adoptando el enfoque de la implementación piso bidimensional (horizontal/vertical) (Durán-Valverde, 2012: 4). Sin embargo, la normativa que regula al Prospera no incluye la ampliación gradual de normas más rigurosas que permitan vislumbrar la inclusión a los trabajadores informales a las prestaciones que caracterizan a los regímenes de seguro social, de conformidad con el Convenio 102 de la OIT sobre la seguridad social.

En segundo lugar, y como se pudo observar en el análisis, se hace evidente la necesidad de mejora en la normativa que dé cabal cumplimiento a los principios y su aplicación en los reglamentos nacionales y que se refleje en los resultados cuantificables y progresivos.

En tercer lugar, los principios más relevantes para la protección de los trabajadores informales, según Prospera, son por supuesto la responsabilidad estatal, la pro- 
tección universal con cobertura horizontal y vertical y el buen gobierno, porque la corrupción inhibe cualquier resultado positivo.

\section{Reconocimientos}

Este artículo es material inédito, cuya base sirvió para preparar la exposición sobre los pisos de protección social en México: «Case of study: Oportunidades, México», International Workshop: The ILO Recommendation on Social Protection Floors: Basic Principles for Innovative Solutions, Sudáfrica, septiembre de 2016.

\section{Referencias}

Bertranou, Fabio (2010). «Iniciativa del piso de protección social». Disponible en http://bit.ly/2I1F2hn.

Camara de diputados (2018). Ley General de Desarrollo Social. Ciudad de México. Congreso de la Union. Disponible en http://bit.ly/2LHZ $7 f_{5}$.

Centro de Estudios Sociales y de Opinión Pública (2014). Integración y concatenación de padrones de beneficiarios como factores de transparencia y rendición de cuentas. Ciudad de México: Cámara de Diputados.

Coneval, Consejo Nacional de Evaluación de la Política de Desarrollo Social (2016). Construcción de las líneas de bienestar, documento metodológico. Ciudad de México. Disponible en http://bit.ly/2JWmDoB.

Durán-VAlverde, Fabio (2012). Articulando los programas de protección social en el contexto de la construcción de los pisos de protección social. Brasilia: AISS.

García Máynez, Eduardo (2008). Introducción al estudio del derecho. Ciudad de México: Porrúa.

Herrera Tapia, Francisco (2009). «Apuntes sobre las instituciones y los programas de desarrollo rural en México: Del Estado benefactor al Estado neoliberal». Estudios Sociales, 17 (33): 9-39. Disponible en http://bit.ly/2Xm9h82.

IMSS, Instituto Mexicano del Seguro Social (2015). Informe financiero y actuarial del IMSS 2015. Ciudad de México. Disponible en http://bit.ly/2HVBMEj.

Moctezuma Navarro, David, José Narro Robles y Lourdes Orozco Hernández (2014). «La mujer en México: Inequidad, pobreza y violencia». Revista Mexicana de Ciencias Políticas y Sociales, 59 (220): 117-146. DOI: 10.1016/So185-1918(14)70803-7. Murayama Rendón, Ciro y Rosa Gómez Tovar (2015). El Mercado de trabajo en México. La opinión social sobre la precariedad laboral. Ciudad de México: UNAM.

NACiOnes Unidas (2016). «The global financial crisis and its impact in the work of the UN system». Disponible en http://bit.ly/2XxIhCJ.

Schwarzer, Hemult y Pablo Casalí (2010). «El piso de protección social: Desarrollo conceptual y aplicación en América Latina». Panorama Laboral 2010. Ciudad de Panamá: OIT. 


\section{Sobre los autores}

Gabriela Mendizábal es profesora e investigadora de la Facultad de Derecho y Ciencias Sociales de la Universidad Autónoma del Estado de Morelos, México. Su correo electrónico es gabymendizabal@yahoo.com.mx.

Patricia Kurczyn es profesora del Instituto de Investigaciones Jurídicas de la Universidad Nacional Autónoma de México. Directora de la Revista Latinoamericana de Derecho Social. Su correo electrónico es patriciakurczyn@yahoo.es. 
La Revista Chilena de Derecho del Trabajo y de la Seguridad Social es una publicación semestral del Departamento de Derecho del Trabajo y de la Seguridad Social de la Facultad de Derecho de la Universidad de Chile, y que tiene por objetivo el análisis dogmático y científico de las instituciones jurídico-laborales y de seguridad social tanto nacionales como del derecho comparado y sus principales efectos en las sociedades en las que rigen.

\author{
DIRECTOR \\ Luis Lizama Portal \\ EDITOR \\ Claudio Palavecino Cáceres \\ SECRETARIO DE REDACCIÓN \\ Eduardo Yañez Monje \\ SITIO WEB \\ revistatrabajo.uchile.cl \\ CORREO ELECTRÓNICO \\ pyanez@derecho.uchile.cl \\ LICENCIA DE ESTE ARTÍCULO \\ Creative Commons Atribución Compartir Igual 4.o Internacional
}

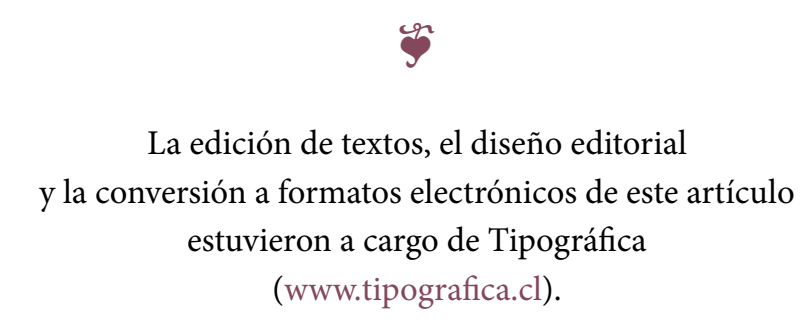

